\title{
Electronic structure of palladium and its relation to uv spectroscopy
}

\section{Christensen, N.E.}

\section{Published in:}

Physical Review B

Link to article, DOI:

10.1103/PhysRevB.14.3446

Publication date:

1976

\section{Document Version}

Publisher's PDF, also known as Version of record

Link back to DTU Orbit

Citation (APA):

Christensen, N. E. (1976). Electronic structure of palladium and its relation to uv spectroscopy. Physical Review B, 14(8), 3446-3461. https://doi.org/10.1103/PhysRevB.14.3446

\section{General rights}

Copyright and moral rights for the publications made accessible in the public portal are retained by the authors and/or other copyright owners and it is a condition of accessing publications that users recognise and abide by the legal requirements associated with these rights.

- Users may download and print one copy of any publication from the public portal for the purpose of private study or research.

- You may not further distribute the material or use it for any profit-making activity or commercial gain

- You may freely distribute the URL identifying the publication in the public portal

If you believe that this document breaches copyright please contact us providing details, and we will remove access to the work immediately and investigate your claim 


\title{
Electronic structure of palladium and its relation to uv spectroscopy
}

\author{
N. E. Christensen \\ Physics Laboratory I, The Technical University of Denmark, DK-2800 Lyngby, Denmark
}

(Received 23 February 1976)

\begin{abstract}
The electronic-energy-band structure of palladium has been calculated by means of the relativistic augmentedplane-wave method covering energies up to $30 \mathrm{eV}$ above the Fermi level. The optical interband transitions producing structure in the dielectric function up to photon energies of $25 \mathrm{eV}$ have been identified. Also the photoemission spectra for polycrystalline samples can be interpreted in terms of the present band model, and the regions in $\overrightarrow{\mathbf{k}}$ space contributing to various elements of structure have been traced out.
\end{abstract}

\section{INTRODUCTION}

$A b$ initio calculations of the electronic band structure of palladium have been reported previously by Mueller et al. ${ }^{1}$ and by Andersen. ${ }^{2}$ Whereas the work of Ref. 1 was based on the nonrelativistic augmented plane wave (APW) method in conjunction with a combined interpolation scheme $\mathrm{e}^{3}$ (also including spin-orbit coupling) Andersen ${ }^{2}$ applied the fully relativistic augmented plane wave (RAPW) method. Fermi-surface properties represented the main object of the calculations presented in Refs. 1 and 2, and thus little attention was paid to the energy bands far above the Fermi level $\left(E_{F}\right)$. The aim of the present work is to examine the optical properties of $\mathrm{Pd}$ such as they manifest themselves in experimental recordings of absorption spectra and photoemission spectra in the ultraviolet (uv) regime. Therefore we needed to extend the previous calculations to include energy levels up to $30 \mathrm{eV}$ above $E_{F}$. This was done by means of the RAPW method using the same muffin-tin potential as in Ref. 2 .

Experimentally, the optical-absorption profile of $\mathrm{Pd}$ has been studied very extensively. In spite of the great amount of experimental results surprisingly little can be extracted about the electronic structure. The disagreement in the published optical data is slightly overwhelming, but is probably due to the difficulty in cleaning ${ }^{4.5}$ the samples and preventing the contamination of virtually clean surfaces. Also aging effects appear to be significant. Weaver ${ }^{6}$ recently compared several experimental results, ${ }^{4,5,7-11}$ and further gave some interpretation in terms of combined interpolationscheme $\mathrm{e}^{3,12}$ band-structure calculations. ${ }^{13-15} \mathrm{Al}-$ though this type of interpolation scheme can be adjusted to reproduce well the band structure in the $d$-band regime and a few electron volts above $E_{F}$, it fails when higher-energy levels (above $10 \mathrm{eV}$ above $E_{F}$ ) are considered. ${ }^{16}$ Therefore, an attempt to relate the optical experiments at photon energies exceeding $\sim 10 \mathrm{eV}$ to a band model must rely on a more exact calculation, such as obtained by the RAPW method, for instance.

Measurements of photoelectron energy spectra have been described by several authors, and some of the most definitive results are probably those presented by Janak, Eastman, and Williams ${ }^{17}$ and by Traum and Smith. ${ }^{14}$ The work described in Ref. 17 includes experimental photoemission spectra for photon energies ranging from 7.7 to $11.6 \mathrm{eV}$ as well as theoretical calculations of the spectra within the direct transition model. The energy bands were obtained by means of the nonrelativistic Korringa-Kohn-Rostoker ${ }^{18}$ method. The experimental work of Ref. 14 represents a part of a systematic study ${ }^{13-15}$ of the photoemission spectra from fcc $d$-band metals and the relation of these spectra to band-structure models.

The main body of the present paper consists of three Secs. II-IV. The band-structure calculation is described in Sec. II. Since the present band calculation is an extension of the one obtained by Andersen, ${ }^{2}$ the results concerning the Fermi surface which can be deduced are identical to those described in Ref. 2, and therefore they will not be considered here. The interband contribution to the imaginary part of the dielectric function is shown in Sec. III, and an attempt to interpret optical experiments from this theoretical profile and partial joint density-of-states functions is presented. Section IV contains the calculated photoemission spectra and a discussion of their relation to the experiments.

\section{Pd BAND STRUCTURE}

The crystal potential which was used in the present calculations is identical to the one used by Andersen. ${ }^{2}$ It is constructed from relativistic Dirac-Slater atomic charge densities ${ }^{19}$ calculated 
for the $4 d^{10}$ configuration. The lattice constant is $a=7.3508$ a.u., the radius in the muff in-tin sphere $R_{S}=2.5857$ a.u., and the Wigner-Seitz radius is $S=2.8727$ a.u. The constant potential between the muff in-tin spheres is $V_{\mathrm{MTZ}}=-1.0294 \mathrm{Ry}$. We have not tried to incorporate shifts in the band structure due to non-muffin-tin effects. The neglect of these corrections is justified by the calculations by Painter et al. ${ }^{20}$ who found that the shifts in Pd were small, a few mRy, for the majority of the energy levels, the most serious corrections being 0.010 and $-0.012 \mathrm{Ry}$ at the $s d$-like $X_{1}$ and the $p$-like $X_{4}$, respectively. The standard RAPW calculation applied here thus follows the lines of previous calculations. ${ }^{2,21}$

Twelve eigenvalues were calculated at $89 \overrightarrow{\mathrm{k}}$ points uniformly distributed over the irreducible $\frac{1}{48}$ Brillouin zone. The results are listed in Table $\mathrm{I}$, and the band structure along symmetry lines is shown in Fig. 1. Characteristic energy gaps are compared to the results obtained by Mueller et al. ${ }^{1}$ in Table II. The eigenvalues were interpolated onto a finer $\vec{k}$ mesh by means of a secondorder interpolation scheme, ${ }^{21}$ and using the GilatRaubenheimer ${ }^{22}$ integration procedure the densityof -states function $N(E)$ was calculated. This function is shown in Figs. 2(a) and 2(b). Critical points are indicated by the symmetry-point label and level number, the levels being counted from below in energy. The Fermi level which was found by integrating $N(E)$ is $E_{F}=0.558 \mathrm{Ry}$, and $N\left(E_{F}\right)=32.1$ (states/atom)/Ry. These values agree with those obtained by Andersen ${ }^{2}$ by means of constant-energy surface integrations.

The spin-orbit splitting $\Delta E_{\mathrm{SO}}$ as obtained in the RAPW calculation is $0.022 \mathrm{Ry}$ at $\Gamma\left(\Gamma_{25}^{\prime} \rightarrow \Gamma_{8}^{+}+\Gamma_{7}^{+}\right)$ and $X\left(X_{5} \rightarrow X_{6}^{+}+X_{7}^{+}\right)$。 In fact one would expect these numbers to differ since $\Delta E_{S O} \simeq \frac{3}{2} \xi_{4 d}$ at $\Gamma$ and $\Delta E_{\mathrm{SO}} \simeq \xi_{4 d}$ at $X$. The value of $\xi_{4 d}$ evaluated at $E=E\left(\Gamma_{25}^{\prime}\right)$ thus becomes $0.014 \mathrm{Ry}$ whereas the value at the top of the $d$-band is larger, namely 0.022 Ry. The apparent SO parameter increases when the energy is varied from the bottom to the top of the $d$-band. ${ }^{2,16}$ The atomic spin-orbit parameter for the $4 d$-states is ${ }^{23} 0.015 \mathrm{Ry}$, and thus very close to the values near the center of the $d$ band.

\section{OPTICAL INTERBAND TRANSITIONS}

Recently Rijsenbrij and Fondse ${ }^{24}$ concluded from a nonrelativistic Korringa-Kohn Rostoker calculation on silver, that it is not necessary to include relativistic effects when the band structure is compared to optical measurements. Apparently their results, as well as an earlier APW calculation $^{25}$ for $\mathrm{Ag}$, agreed better with the majority of optical measurements, in particular, concerning the gap $L_{1}-L_{2}^{\prime}$, than did the RAPW calculation. ${ }^{26}$ However, this does not mean that relativistic effects should be excluded, but rather it indicates that slight corrections to the crystal potential (non-muffin-tin terms) are necessary to obtain a correct value of this gap. The fact that the RAPW results differ from the APW results in the case of silver, and thus also for Pd, by up to $1 \mathrm{eV} \mathrm{de-}$ finitively indicates that relativistic effects are important when the band model is checked against optical measurements where the accuracy often is $\approx 0.1 \mathrm{eV}$. Therefore, we shall here only consider the relativistic band models when comparing to optical experiments.

The partial joint density-of-states function corresponding to the initial band $i$ and final band $f$ is defined through

$$
\begin{aligned}
J_{i f}(\hbar \omega)=\frac{2 \Omega}{(2 \pi)^{3}} \int & d \overrightarrow{\mathrm{k}} \delta\left(E_{F}(\overrightarrow{\mathrm{k}})-E_{i}(\overrightarrow{\mathrm{k}})-\hbar \omega\right) \\
& \times f\left(E_{\boldsymbol{i}}(\overrightarrow{\mathrm{k}})\right)\left[1-f\left(E_{f}(\overrightarrow{\mathrm{k}})\right)\right] .
\end{aligned}
$$

This function is a measure of the number of transitions between states $i$ and $f$ separated in energy by $\hbar \omega$. The Fermi functions $f\left(E_{i}(\vec{k})\right)$ and $\left[1-f\left(E_{f}(\overrightarrow{\mathrm{k}})\right)\right]$ ensure that only occupied initial states and empty final states are included. The total joint density of states $J(\hbar \omega)$ is obtained by summing over all $i$ and $f$ :

$$
J(\hbar \omega)=\sum_{i, f} J_{i f}(\hbar \omega) .
$$

If the optical transition matrix elements could be considered as independent of energy and wave number, this function (2) would be proportional to the interband part of the imaginary part $\epsilon_{2}(\omega)$ of the dielectric function multiplied by $\omega^{2}$. Experimentally the interband contribution to $\epsilon_{2}(\omega)$ is obtained by static reflectance measurements. Although modulation spectroscopy offers a greater opportunity to identify transitions at specific points in $\overrightarrow{\mathrm{k}}$ space, such as critical points, no such results have been presented for Pd. We therefore must rely on static experimental $\epsilon_{2}$ profiles in our interpretation and realize that critical transitions may easily be masked by a large noncritical background, which even may contain structure due to varying matrix elements.

Some of the experimental observations may be briefly summarized by quoting a few spectral positions of "structure" in the reflectivity of $\mathrm{Pd}$. Duis ebaeva et $a l .^{7}$ reported structure at $0.5 \mathrm{eV}$, Lostis $^{10}$ at $1.1 \mathrm{eV}$, Johnson and Christy ${ }^{11}$ found a change in slope at $1.4 \mathrm{eV}$, minima near 2.4 and $4.5 \mathrm{eV}$, whereas Vehse et al. ${ }^{4}$ reported a minimum at $4 \mathrm{eV}$ and the measurements by $\mathrm{Yu}$ and Spicer ${ }^{5}$ 
TABLE I. Energy eigenvalues at $89 \overrightarrow{\mathrm{k}}$ points as obtained by the RAPW method. The energies are measured in mRy from the muffin-tin zero $\left(V_{\mathrm{MTZ}}=-1.0294 \mathrm{Ry}\right)$.

\begin{tabular}{|c|c|c|c|c|c|c|c|c|c|c|c|c|c|}
\hline & $\overrightarrow{\mathrm{k}} / \frac{\pi}{4 a}$ & $\begin{array}{c}\text { Band } \\
1\end{array}$ & $\begin{array}{c}\text { Band } \\
2\end{array}$ & $\begin{array}{c}\text { Band } \\
3\end{array}$ & $\begin{array}{c}\text { Band } \\
4\end{array}$ & $\begin{array}{c}\text { Band } \\
5\end{array}$ & $\begin{array}{c}\text { Band } \\
6\end{array}$ & $\begin{array}{c}\text { Band } \\
7\end{array}$ & $\begin{array}{c}\text { Band } \\
8\end{array}$ & $\begin{array}{c}\text { Band } \\
9\end{array}$ & $\begin{array}{c}\text { Band } \\
10\end{array}$ & $\begin{array}{c}\text { Band } \\
11\end{array}$ & $\begin{array}{c}\text { Band } \\
12\end{array}$ \\
\hline$\Gamma$ & 000 & 43 & 353 & 353 & 375 & 472 & 472 & 1860 & 2150 & 2162 & 2162 & 2508 & 2800 \\
\hline$\Delta$ & $\begin{array}{lll}0 & 1 & 0\end{array}$ & 57 & 347 & 356 & 378 & 464 & 475 & 1874 & 2104 & 2147 & 2161 & 2605 & 2732 \\
\hline$\Delta$ & 020 & 96 & 330 & 372 & 393 & 445 & 483 & 1913 & 2015 & 2100 & 2123 & 2652 & 2669 \\
\hline$\Delta$ & 030 & 152 & 303 & 393 & 420 & 432 & 495 & 1943 & 1967 & 2002 & 2033 & 2515 & 2657 \\
\hline$\Delta$ & 040 & 198 & 273 & 419 & 454 & 457 & 511 & $1864^{\circ}$ & 1882 & 1906 & 2026 & 2290 & 2730 \\
\hline$\Delta$ & 050 & 203 & 244 & 478 & 497 & 513 & 520 & 1731 & 1750 & 1770 & 2080 & 2147 & 2860 \\
\hline$\Delta$ & 060 & 185 & 221 & 525 & 529 & 553 & 630 & 1581 & 1635 & 1663 & 2103 & 2122 & 2947 \\
\hline$\Delta$ & 070 & 168 & 206 & 543 & 557 & 580 & 759 & 1414 & 1553 & 1587 & 2102 & 2148 & 3219 \\
\hline$X$ & 080 & 161 & 201 & 547 & 570 & 591 & 825 & 1333 & 1525 & 1560 & 2106 & 2157 & 2879 \\
\hline \multirow[t]{7}{*}{$\Sigma$} & 110 & 70 & 345 & 359 & 381 & 461 & 473 & 1884 & 2020 & 2146 & 2215 & 2575 & 2735 \\
\hline & 120 & 108 & 330 & 370 & 395 & 447 & 483 & 1905 & 1924 & 2117 & 2215 & 2517 & 2683 \\
\hline & 130 & 163 & 305 & 382 & 418 & 443 & 500 & 1842 & 1905 & 2076 & 2108 & 2513 & 2623 \\
\hline & 140 & 208 & 277 & 390 & 452 & 473 & 526 & 1765 & 1830 & 1955 & 2085 & 2356 & 2669 \\
\hline & 150 & 213 & 250 & 429 & 493 & 514 & 580 & 1675 & 1721 & 1785 & 2119 & 2229 & 2778 \\
\hline & 160 & 194 & 228 & 477 & 530 & 543 & 683 & 1573 & 1605 & 1624 & 2150 & 2179 & 2854 \\
\hline & 170 & 177 & 214 & 514 & 544 & 571 & 820 & 1416 & 1523 & 1553 & 2155 & 2175 & 2858 \\
\hline$Z$ & 180 & 170 & 209 & 531 & 544 & 583 & 874 & 1332 & 1497 & 1528 & 2165 & 2170 & 2851 \\
\hline \multirow[t]{6}{*}{$\Sigma$} & 220 & 143 & 325 & 372 & 402 & 440 & 492 & 1817 & 1878 & 2134 & 2328 & 2352 & 2736 \\
\hline & 230 & 192 & 310 & 369 & 419 & 437 & 530 & 1728 & 1818 & 2148 & 2165 & 2464 & 2636 \\
\hline & 240 & 236 & 288 & 357 & 450 & 462 & 580 & 1649 & 1730 & 1979 & 2187 & 2455 & 2597 \\
\hline & 250 & 242 & 266 & 371 & 489 & 496 & 657 & 1572 & 1629 & 1799 & 2209 & 2362 & 2679 \\
\hline & 260 & 220 & 248 & 409 & 512 & 536 & 770 & 1501 & 1537 & 1611 & 2226 & 2327 & 2733 \\
\hline & 270 & 201 & 236 & 445 & 516 & 568 & 901 & 1416 & 1460 & 1480 & 2236 & 2330 & 2749 \\
\hline$Z$ & 280 & 194 & 232 & 462 & 513 & 580 & 978 & 1330 & 1434 & 1458 & 2240 & 2336 & 2749 \\
\hline \multirow[t]{5}{*}{$\Sigma$} & 330 & 233 & 310 & 361 & 419 & 434 & 581 & 1631 & 1723 & 2106 & 2240 & 2385 & 2730 \\
\hline & 340 & 270 & 303 & 338 & 432 & 458 & 647 & 1550 & 1621 & 1993 & 2299 & 2326 & 2715 \\
\hline & 350 & 279 & 292 & 324 & 461 & 495 & 738 & 1481 & 1520 & 1831 & 2316 & 2329 & 2770 \\
\hline & 360 & 253 & 281 & 349 & 480 & 534 & 859 & 1430 & 1450 & 1643 & 2335 & 2364 & 2752 \\
\hline & 370 & 230 & 273 & 375 & 482 & 567 & 1002 & 1362 & 1392 & 1463 & 2335 & 2451 & 2680 \\
\hline$Z$ & 380 & 223 & 270 & 388 & 479 & 579 & 1098 & 1329 & 1342 & 1400 & 2335 & 2533 & 2603 \\
\hline \multirow[t]{4}{*}{$\Sigma$} & 440 & 286 & 314 & 333 & 420 & 473 & 726 & 1472 & 1512 & 1999 & 2170 & 2409 & 2684 \\
\hline & 450 & 286 & 309 & 327 & 439 & 500 & 824 & 1410 & 1425 & 1888 & 2151 & 2469 & 2736 \\
\hline & 460 & 266 & 308 & 326 & 457 & 535 & 949 & 1312 & 1379 & 1696 & 2241 & 2471 & 2921 \\
\hline & 470 & 245 & 315 & 326 & 462 & 566 & 1098 & 1247 & 1357 & 1501 & 2366 & 2463 & 2764 \\
\hline$W$ & 480 & 237 & 318 & 328 & 462 & 579 & 1209 & 1237 & 1331 & 1381 & 2458 & 2460 & 2658 \\
\hline \multirow[t]{3}{*}{$\Sigma$} & 550 & 260 & 308 & 355 & 444 & 514 & 925 & 1294 & 1374 & 1939 & 1997 & 2615 & 2701 \\
\hline & 560 & 241 & 289 & 375 & 463 & 539 & 1044 & 1197 & 1351 & 1754 & 2101 & 2629 & 2805 \\
\hline & 570 & 228 & 274 & 385 & 475 & 567 & 1124 & 1193 & 1340 & 1558 & 2238 & 2610 & 2640 \\
\hline \multirow[t]{8}{*}{$K, U$} & 660 & 215 & 262 & 423 & 486 & 549 & 1090 & 1157 & 1338 & 1738 & 2052 & 2626 & 2757 \\
\hline & 111 & 83 & 344 & 360 & 384 & 463 & 470 & 1882 & 1961 & 2190 & 2210 & 2547 & 2699 \\
\hline & 121 & 119 & 332 & 369 & 395 & 452 & 481 & 1830 & 1934 & 2159 & 2245 & 2495 & 2614 \\
\hline & 131 & 171 & 311 & 376 & 410 & 459 & 500 & 1747 & 1942 & 2057 & 2180 & 2497 & 2612 \\
\hline & 141 & 214 & 285 & 379 & 432 & 506 & 526 & 1661 & 1903 & 1928 & 2101 & 2417 & 2677 \\
\hline & 151 & 219 & 258 & 412 & 463 & 542 & 599 & 1569 & 1771 & 1802 & 2079 & 2333 & 2768 \\
\hline & 161 & 202 & 236 & 460 & 496 & 560 & 746 & 1482 & 1596 & 1692 & 2089 & 2302 & 2830 \\
\hline & 171 & 184 & 222 & 497 & 521 & 571 & 844 & 1405 & 1437 & 1621 & 2105 & 2297 & 2839 \\
\hline \multirow[t]{12}{*}{$S$} & 181 & 178 & 217 & 512 & 531 & 574 & 916 & 1331 & 1401 & 1597 & 2113 & 2297 & 2836 \\
\hline & 221 & 150 & 333 & 368 & 398 & 452 & 492 & 1727 & 1919 & 2173 & 2347 & 2374 & 2588 \\
\hline & 231 & 192 & 325 & 366 & 403 & 463 & 524 & 1630 & 1886 & 2103 & 2253 & 2475 & 2534 \\
\hline & 241 & 227 & 309 & 356 & 420 & 501 & 575 & 1538 & 1829 & 1970 & 2169 & 2508 & 2593 \\
\hline & 251 & 235 & 282 & 372 & 448 & 536 & 662 & 1448 & 1748 & 1809 & 2122 & 2476 & 2682 \\
\hline & 261 & 221 & 258 & 406 & 479 & 555 & 787 & 1367 & 1611 & 1683 & 2118 & 2457 & 2735 \\
\hline & 271 & 205 & 244 & 438 & 500 & 565 & 926 & 1308 & 1434 & 1626 & 2131 & 2455 & 2754 \\
\hline & 281 & 199 & 240 & 453 & 506 & 568 & 1010 & 1287 & 1337 & 1607 & 2139 & 2457 & 2755 \\
\hline & $\begin{array}{lll}3 & 31\end{array}$ & 218 & 338 & 362 & 398 & 462 & 574 & 1525 & 1813 & 2074 & 2319 & 2378 & 2559 \\
\hline & 341 & 241 & 336 & 347 & 409 & 487 & 642 & 1429 & 1740 & 1979 & 2245 & 2410 & 2643 \\
\hline & 351 & 254 & 306 & 347 & 465 & 521 & 738 & 1342 & 1669 & 1832 & 2193 & 2459 & 2754 \\
\hline & 361 & 250 & 283 & 359 & 460 & 545 & 868 & 1266 & 1607 & 1650 & 2200 & 2495 & 2777 \\
\hline
\end{tabular}


TABLE I. (continued).

\begin{tabular}{|c|c|c|c|c|c|c|c|c|c|c|c|c|c|}
\hline & $\overrightarrow{\mathrm{k}} / \frac{\pi}{4 a}$ & $\begin{array}{c}\text { Band } \\
1\end{array}$ & $\begin{array}{c}\text { Band } \\
2\end{array}$ & $\begin{array}{c}\text { Band } \\
3\end{array}$ & $\begin{array}{c}\text { Band } \\
4\end{array}$ & $\begin{array}{c}\text { Band } \\
5\end{array}$ & $\begin{array}{c}\text { Band } \\
6\end{array}$ & $\begin{array}{c}\text { Band } \\
7\end{array}$ & $\begin{array}{c}\text { Band } \\
8\end{array}$ & $\begin{array}{c}\text { Band } \\
9\end{array}$ & $\begin{array}{c}\text { Band } \\
10\end{array}$ & $\begin{array}{c}\text { Band } \\
11\end{array}$ & $\begin{array}{c}\text { Band } \\
12\end{array}$ \\
\hline & 371 & 234 & 276 & 376 & 473 & 560 & 1019 & 1213 & 1458 & 1572 & 2226 & 2545 & 2713 \\
\hline & 381 & 228 & 274 & 385 & 475 & 566 & 1124 & 1193 & 1340 & 1558 & 2239 & 2610 & 2640 \\
\hline & 441 & 253 & 324 & 370 & 404 & 493 & 721 & 1334 & 1656 & 1978 & 2165 & 2449 & 2652 \\
\hline & 451 & 265 & 302 & 364 & 422 & 516 & 822 & 1251 & 1586 & 1885 & 2127 & 2500 & 2719 \\
\hline & 461 & 271 & 284 & 352 & 444 & 541 & 950 & 1180 & 1535 & 1702 & 2198 & 2497 & 2859 \\
\hline \multirow[t]{9}{*}{$Q$} & 471 & 252 & 301 & 339 & 458 & 559 & 1087 & 1146 & 1489 & 1523 & 2301 & 2481 & 2830 \\
\hline & 551 & 256 & 300 & 373 & 429 & 527 & 918 & 1171 & 1521 & 1946 & 1982 & 2626 & 2687 \\
\hline & 561 & 244 & 287 & 376 & 451 & 545 & 1019 & 1120 & 1480 & 1764 & 2088 & 2617 & 2806 \\
\hline & 222 & 169 & 354 & 363 & 394 & 474 & 490 & 1613 & 1911 & 2277 & 2295 & 2469 & 2499 \\
\hline & 232 & 193 & 357 & 369 & 390 & 496 & 516 & 1507 & 1884 & 2149 & 2314 & 2434 & 2527 \\
\hline & 242 & 21.6 & 340 & 365 & 399 & 537 & 575 & 1409 & 1855 & 2010 & 2151 & 2578 & 2593 \\
\hline & 252 & 229 & 307 & 375 & 418 & 556 & 689 & 1318 & 1783 & 1895 & 2040 & 2630 & 2650 \\
\hline & 262 & 229 & 279 & 394 & 445 & 562 & 831 & 1238 & 1627 & 1809 & 2024 & 2635 & 2718 \\
\hline & 272 & 220 & 266 & 414 & 472 & 556 & 986 & 1181 & 1448 & 1756 & 2042 & 2625 & 2749 \\
\hline \multirow[t]{8}{*}{$U, K$} & 282 & 215 & 262 & 422 & 486 & 549 & 1090 & 1157 & 1338 & 1738 & 2052 & 2626 & 2758 \\
\hline & 332 & 200 & 353 & 382 & 411 & 498 & 554 & 1396 & 1846 & 2118 & 2314 & 2447 & 2492 \\
\hline & 342 & 213 & 341 & 380 & 415 & 526 & 626 & 1298 & 1814 & 2011 & 2191 & 2514 & 2585 \\
\hline & 352 & 229 & 317 & 385 & 411 & 549 & 740 & 1212 & 1775 & 1887 & 2089 & 2566 & 2701 \\
\hline & 362 & 243 & 294 & 381 & 428 & 553 & 882 & 1143 & 1659 & 1797 & 2069 & 2596 & 2777 \\
\hline & 372 & 244 & 287 & 376 & 452 & 545 & 1019 & 1120 & 1480 & 1764 & 2089 & 2617 & 2806 \\
\hline & 442 & 217 & 333 & 379 & 437 & 523 & 702 & 1206 & 1772 & 1977 & 2152 & 2525 & 2599 \\
\hline & 452 & 231 & 315 & 389 & 421 & 540 & 803 & 1135 & 1735 & 1898 & 2091 & 2549 & 2680 \\
\hline \multirow[t]{2}{*}{$Q$} & 462 & 253 & 292 & 383 & 421 & 546 & 909 & 1103 & 1695 & 1746 & 2126 & 2533 & 2787 \\
\hline & 552 & 236 & 308 & 394 & 415 & 547 & 862 & 1099 & 1690 & 1957 & 1978 & 2630 & 2650 \\
\hline \multirow[t]{5}{*}{$\Lambda$} & 333 & 192 & 347 & 375 & 491 & 525 & 537 & 1285 & 1817 & 2183 & 2215 & 2510 & 2530 \\
\hline & 343 & 197 & 341 & 371 & 480 & 550 & 618 & 1194 & 1798 & 2091 & 2150 & 2549 & 2564 \\
\hline & 353 & 213 & 325 & 378 & 444 & 557 & 742 & 1125 & 1782 & 2004 & 2034 & 2602 & 2630 \\
\hline & 363 & 236 & 308 & 394 & 415 & 547 & 862 & 1099 & 1690 & 1957 & 1978 & 2630 & 2650 \\
\hline & 443 & 195 & 339 & 367 & 507 & 547 & 665 & 1123 & 1783 & 2066 & 2139 & 2556 & 2569 \\
\hline$Q$ & 453 & 207 & 330 & 372 & 468 & 552 & 744 & 1095 & 1780 & 1991 & 2088 & 2567 & 2626 \\
\hline$L$ & 444 & 188 & 339 & 365 & 548 & 562 & 628 & 1095 & 1774 & 2112 & 2146 & 2550 & 2558 \\
\hline
\end{tabular}

revealed shoulders near 3 and $6 \mathrm{eV}$.

Three sets of experimental data have been selected, and the derived $\epsilon_{2}$ functions are shown in Fig. 4. Curve (a), by Vehse et al.,$^{4}$ extends to high photon energies and was based on measurements on films in situ. The $\epsilon_{2}$ profile presented by Johnson and Christy ${ }^{11}$ was obtained from measurements in $N_{2}$. Weaver ${ }^{27}$ has succeeded in reproducing these data reasonably well, and also the observation by Duisebaeva ${ }^{7}$ [ curve (c) in Fig. 3] concerning the peak at $\approx 0.5$ has been confirmed. ${ }^{27}$ In the same figure (Fig. 3) is a theoretical curve marked RAPW. It represents the joint density of states divided by $\omega^{2}$ as calculated from the present RAPW band-structure calculation. The theoretical curve has been scaled to the experiment of Ref. 11 at $2.5 \mathrm{eV}$.
The part of the spectrum lying between 3 and 8 $\mathrm{eV}$ is probably the part which is most easy to relate to the band structure. Clearly the photon energy in this regime is so large that it is beyond the region where the intraband contributions can be essential, these parts being very difficult to subtract in the case of $\mathrm{Pd}$ where the interband transitions are important even for photon energies very close to 0 . On the other hand the photon energy range 2-8 eV does not involve very-highlying bands, and thus it covers a regime whe re we can have a fair confidence in the band calculation. We observe three clear changes of slope in curve (a) at $A(3.0 \mathrm{eV}), B(4.4 \mathrm{eV})$, and $C(7.2 \mathrm{eV})$ which could be interband edges. Only the kink $B$ at $4.4 \mathrm{eV}$ is clearly in the experimental curve (c) as well. On the other hand, two edgelike features are seen in 


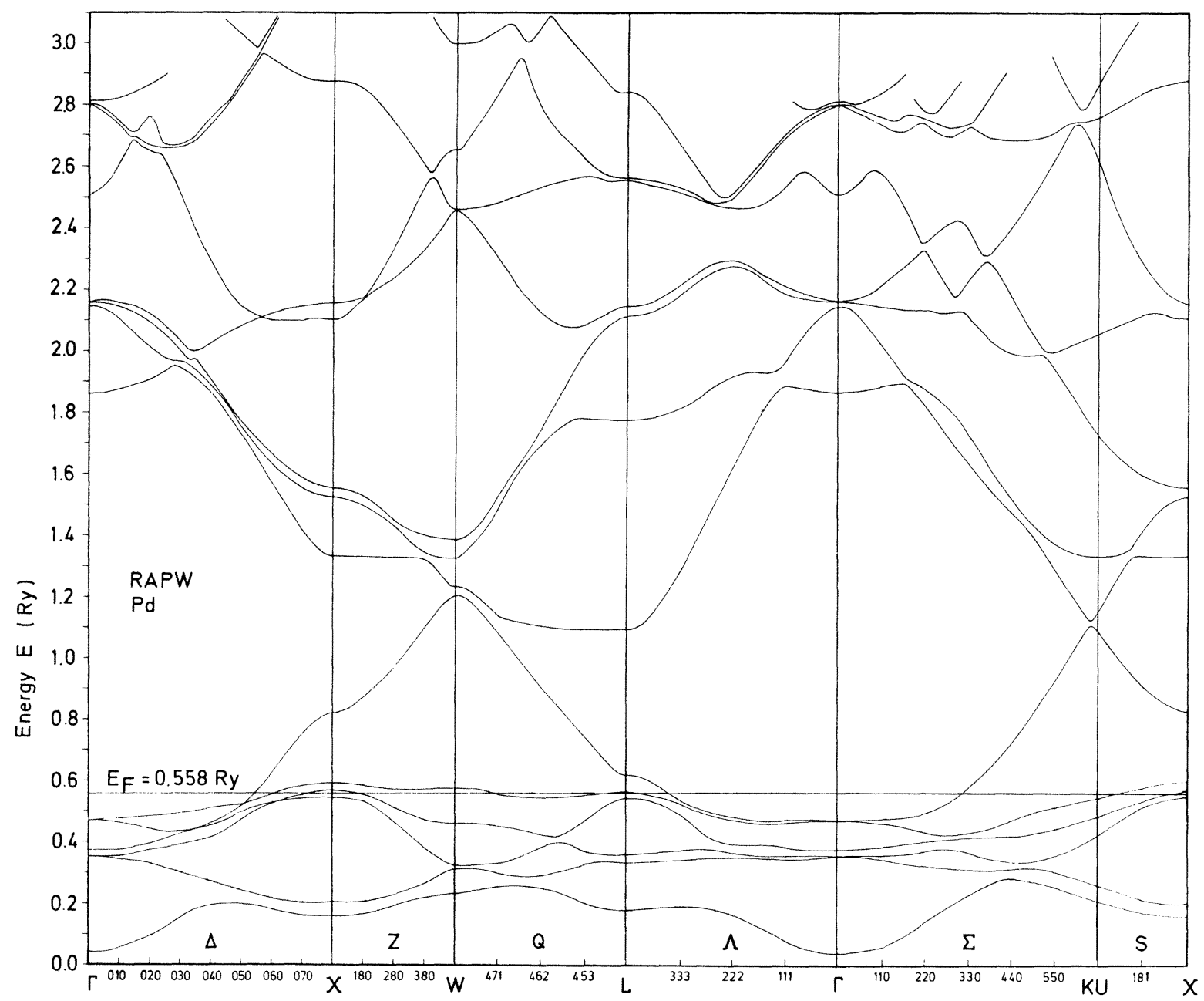

FIG. 1. Band structure of Pd along symmetry lines as calculated by the RAPW method. The fermi level $E_{F}=0.558$ Ry above the muffin-tin zero.

(c) at $3.3 \mathrm{eV}(D)$ and $3.7 \mathrm{eV}(E)$. The kink $A$ cannot be clearly retrieved in the theoretical trace, but $B, C, D$, and $E$ all have clear counterparts in the RAPW spectrum. The big hump near $B$ is strongly exaggerated in the theoretical curve, probably due to the assumed constancy of the matrix elements. Some information about the parts of the band structure which contribute to $\epsilon_{2}(\omega)$ in specific energy ranges may be obtained by examining the individual partial joint density-of-states functions $J_{i f}(\hbar \omega)$ [Eq.(1)]. These functions are shown in Figs. 4(a)-4(f). It is seen that the transitions from band 3 to band 6 become essential near the spec$\operatorname{tral}$ position $A$, whereas $D$ can be related to a $2 \rightarrow 5$ edge. The onset of the $2 \rightarrow 5$ transitions at $3.3 \mathrm{eV}$ takes place near and at the symmetry line $Q$, the final states being at the Fermi level. The $2 \rightarrow 6$ transitions are also important in this regime, but they start at somewhat lower energies. The onset is $2.94 \mathrm{eV}(\mathrm{RAPW})$ at $\Lambda$, the final states being at $E_{F}$. The $3-5$ transitions give rise to an upper edge at $3.5 \mathrm{eV}$ (near $W$ ) which may be related to $E$. Figure 4(a) shows that $J_{15}$ as well as $J_{16}$ have sharp edges at 4.3-4.5 eV which could be related to $B$. These edges are in part due to transitions near $Q(1 \rightarrow 5)$ and $\Sigma(1 \rightarrow 6)$.

The element $C$ at $7.2 \mathrm{eV}$ in the experimental curve (a) is also found in the RAPW curve. This feature is unambiguously identified by means of the partial joint density-of-states curves [Fig. 4(e)] as representing the $5-7$ edge, due to transitions from band $5\left(E_{F}\right)$ to band 7 at the symmetry line $Q$. The fact that this identification appears so safe, and that the agreement between the calcula- 
TABLE II. Comparison of characteristic energy separations from two $a b$ initio calculations, the nonrelativistic APW calculation by Mueller et al. (Ref. 1) and the present RAPW calculation.

\begin{tabular}{|c|c|c|c|c|}
\hline $\begin{array}{l}\text { Double-group } \\
\text { notation }\end{array}$ & $\begin{array}{l}\text { Single-group } \\
\text { notation }\end{array}$ & $\begin{array}{c}\text { Band } \\
\text { indices }\end{array}$ & $\begin{array}{c}\text { Mueller et al. } \\
\text { (Ref. 1) } \\
\text { APW }\end{array}$ & $\begin{array}{c}\text { Present } \\
\text { RAPW }\end{array}$ \\
\hline \multicolumn{5}{|c|}{$s$ bandwidth } \\
\hline$X_{6}^{-}-\Gamma_{6}^{+}$ & $X_{4}^{\prime}-\Gamma_{1}$ & $6 ; 1$ & 0.751 & 0.782 \\
\hline$X_{6}^{-}-L_{4}^{-}$ & $X_{4}^{\prime}-L_{2}^{\prime}$ & $6 ; 6$ & 0.200 & 0.197 \\
\hline$L_{4}^{-}-\Gamma_{6}^{+}$ & $L_{2}^{\prime}-\Gamma_{1}$ & $6 ; 1$ & 0.551 & 0.585 \\
\hline \multicolumn{5}{|c|}{$d$ bandwidth } \\
\hline$X_{7}^{+}-X_{6}^{+}$ & $X_{5}-X_{1}$ & $5 ; 1$ & 0.396 & 0.430 \\
\hline$\Gamma_{8}^{+}-\Gamma_{8}^{+}$ & $\Gamma_{12}-\Gamma_{25}^{\prime}$ & $(6,5) ;(2,3)$ & 0.103 & 0.119 \\
\hline$L_{6+5}^{+}-L_{4}^{+}$ & $L_{3}-L_{1}$ & $5 ; 1$ & 0.336 & 0.375 \\
\hline \multicolumn{5}{|c|}{$s-d$ separation } \\
\hline$L_{4}^{-}-L_{6+5}^{+}$ & $L_{2}^{\prime}-L_{3}$ & $6 ; 5$ & 0.133 & 0.066 \\
\hline$X_{6}^{-}-X_{7}^{+}$ & $X_{4}^{\prime}-X_{5}$ & $6 ; 5$ & 0.308 & 0.234 \\
\hline \multicolumn{5}{|c|}{ "L-gap" } \\
\hline \multirow[t]{2}{*}{$L_{4}^{+}-L_{4}^{-}$} & $L_{1}-L_{2}^{\prime}$ & $7 ; 6$ & & 0.467 \\
\hline & & & Ry & Ry \\
\hline
\end{tabular}

ted gap and the experiment is within $\approx 0.1 \mathrm{eV}$ gives a strong support to the present RAPW band model since level 7 at $L$ is particularly sensitive to the potential.

The experimental $\epsilon_{2}$ function (a) has in the highenergy regime (9-25 eV) maxima near the following spectral positions, 9.7, 12.5, 15.0, 16.2, 17.3, and $20.5 \mathrm{eV}$. As follows from Fig. 3, it is extremely difficult to relate these "peaks" unambiguously to the structure in the total $\epsilon_{2}$ spectrum calculated in the crude constant matrix approximation. However, considering the partial functions $J_{i f}$ in Figs. $4(\mathrm{c})$ and $4(\mathrm{~d})$ it appears that the $3 \rightarrow 7$ and $4-7$ transitions are contributing essentially to the $9.7-\mathrm{eV}$ peak. The sharp peak in $J_{47}$ at $9.3 \mathrm{eV}$ is related to parallel-band transitions from band 4 to band 7 at a $Q$ point. The $3 \rightarrow 7$ transitions at the symmetry point $L$, according to the RAPW calculation [Table I and Fig. 4(c)], give rise to a singular peak at $9.9 \mathrm{eV}$. The structure at $12.5 \mathrm{eV}$ may be related to $1 \rightarrow 7,2 \rightarrow 7$, and $3 \rightarrow 7$ transitions. The onset of the $1 \rightarrow 7$ transitions at $12.3 \mathrm{eV}$ takes place at $L$, whereas $J_{27}$ has a singularity at $W$ at $\hbar \omega=12.5 \mathrm{eV}$. Figure 4 shows that the only band pair which in the present model gives significant structure near $\hbar \omega=15 \mathrm{eV}$ is $(i, f)=(1,8)$. These transitions take place near $Q$ and $Z$ points, the onset (calculated to be $14.9 \mathrm{eV}$ ) taking place at $W$. Figure 4 shows that $J_{58}$ has a very pronounced peak at $\hbar \omega=16.8 \mathrm{eV}$. This corresponds to transi- tions from band 5 at the Fermi level to band 8 in a region around $L$ (including $Q$ as well as $\Lambda$ points). It is suggested that these transitions in the extended region around $L$ are responsible for the structure observed at $17.3 \mathrm{eV}$ in curve (a) in Fig. 3. If this interpretation is correct, one would also expect that the $4-8$ peak at $18.1 \mathrm{eV}$ [Fig. 4(d)] might show up in the experiment. In fact, some structure, although weak, is observed at $18.4 \mathrm{eV}$. The very obvious maximum in the experimental trace (Fig. 3) at $20.5 \mathrm{eV}$ appears to coincide with a minimum in the calculated spectrum. However, as follows from the comments given above to the elements of structure at 17.3 and $18.4 \mathrm{eV}$ we may now be considering an energy range where the RAPW calculation easily can contain errors of several tenths of an eV in magnitude. Further, what probably is more important, the constant matrix element approximation will be extremely bad in this regime since $d \rightarrow f$ transitions with high probability now can occur. According to the RAPW band calculation, the $d \rightarrow f$ transitions at $\Gamma$ start near $19 \mathrm{eV},(5,6) \rightarrow 7$. The $f$ bands in gold ${ }^{28}$ appeared to be placed correctly by the RAPW calculation when compared to modulation spectroscopy and photoemission results, and further, the $4 \rightarrow 7$ transitions $\left(\Gamma_{7}^{+} \rightarrow \Gamma_{6}^{-}\right)$seemed to have particularly strong transition probability. In $\mathrm{Pd}$ the $4-7$ gap at $\Gamma$ is calculated to be $20.2 \mathrm{eV}$ (Table I). This value is in excellent agreement with the ex- 

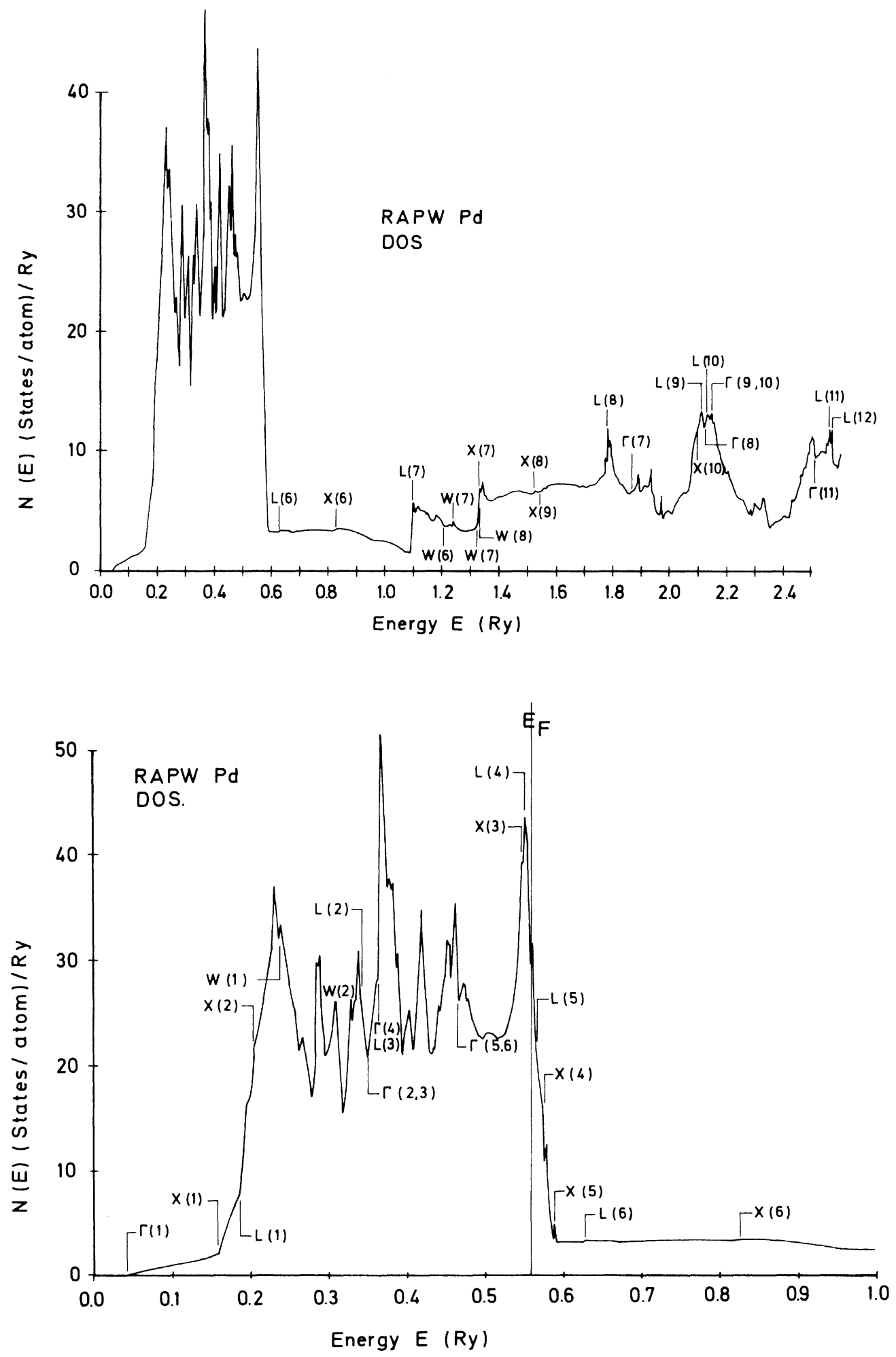

FIG. 2. (a) Density-of-states function over the entire energy range covered by the RAPW calculation. Some critical points are indicated, the numbers being the level numbers. The eigenvalues at each $k$ point is counted from below. (b) Blowup of the DOS in the $d$-band range. 


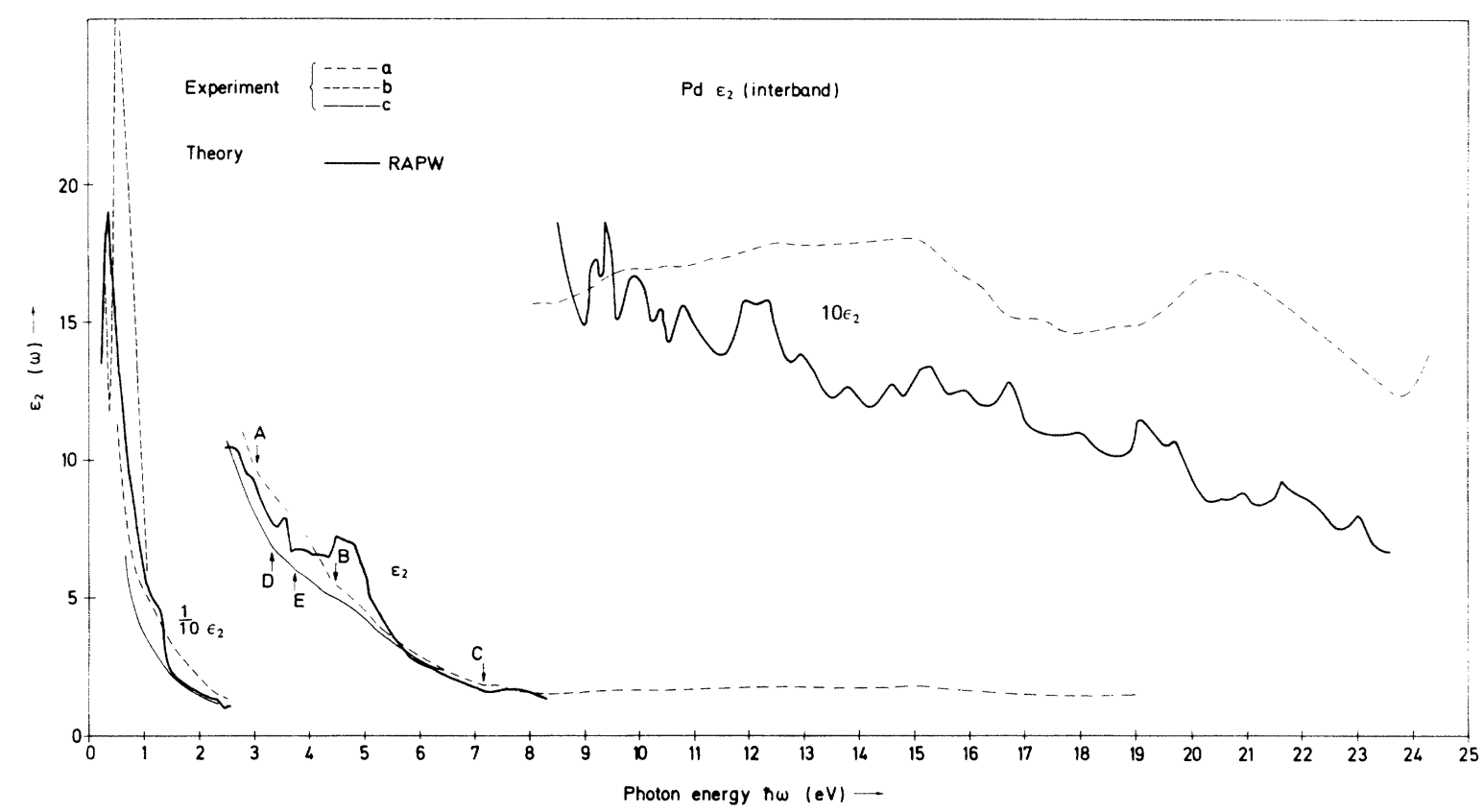

FIG. 3. Interband contribution to the imaginary part $\epsilon_{2}(\omega)$ of the dielectric function as calculated from the joint density of states together with experimental $\epsilon_{2}$ traces. (a)-(c) Experiments by Vehse et al., (a), Ref. 4, Duisebaeva et al . (b), Ref. 7, and Johnson and Cristy (c), Ref. 11.

periment if it is assumed to represent the edge for transitions giving rise to the broad maximum around $20.5 \mathrm{eV}$ in the experimental $\epsilon_{2}$ curve. $^{4}$

In the range of low photon energies, $0-2 \mathrm{eV}$, there are large differences between the experimental results. Although the band calculation must be expected to be very good in this regime, it also follows from Fig. 3 that the theoretical $\epsilon_{2}$ profile differs from the experiments. This again is ascribed to the crude constant matrix element approximation. At very low energies some $d-d$ transitions are included in the calculation although they, due to their forbidden character, cannot be expected to contribute essentially to the spectrum. The structure which is clear in the theoretical curve at $\hbar \omega \approx 1.1 \mathrm{eV}$ may also be noticed in the experimental traces (a) and (c). Further curve (b) exhibits, as mentioned earlier, a strong peak at $\hbar \omega \approx 0.5 \mathrm{eV}$, which also is found in the calculation. In order to analyze the optical properties further for small photon energies Weaver ${ }^{27}$ recently performed new measurements. The results were obtained by using a high purity sample which was etched to remove work damage, annealed in vacuum to promote grain growth, then flashed in ultrahigh vacuum before it was placed in the calorimeter where the absorptivity was measured. The conductivity $\sigma(\omega)$ which was obtained after a
Kramers-Kroenig analysis is shown in Fig. 5 .

It follows from Fig. 5 that the new results by Weaver support the results of Duisebaeva et al. ${ }^{\text {? }}$ concerning the peak at $0.5 \mathrm{eV}$, and also it is clear that a peak does sort out at $1.1 \mathrm{eV}$. It was suggested $d^{27}$ that these two elements of structure essentially are due to $4 \rightarrow 5$ transitions regions around $\Lambda$ and $L$ and this is also very reasonable when compared to the present band models. The comparison to the RAPW result is obscured by the strong $d-d(3 \rightarrow 4$ near $X)$ contribution, and therefore this has been subtracted in one of the curves shown in Fig. 5.

\section{PHOTOEMISSION}

In the present analysis of the photoemission spectra of Pd, we will assume that the experimental results have been obtained from polycrystall ine samples, and that "surface emission" processes $^{29,30}$ contribute negligibly to the spectra compared to bulk processes. Further, it is assumed that the bulk photoemission can be related to the one-electron band model in the manner described by the conventional three-step model, where only direct optical transitions are considered. In this model the photoelectron spectrum $N(E, \hbar \omega)$ can be expressed as 

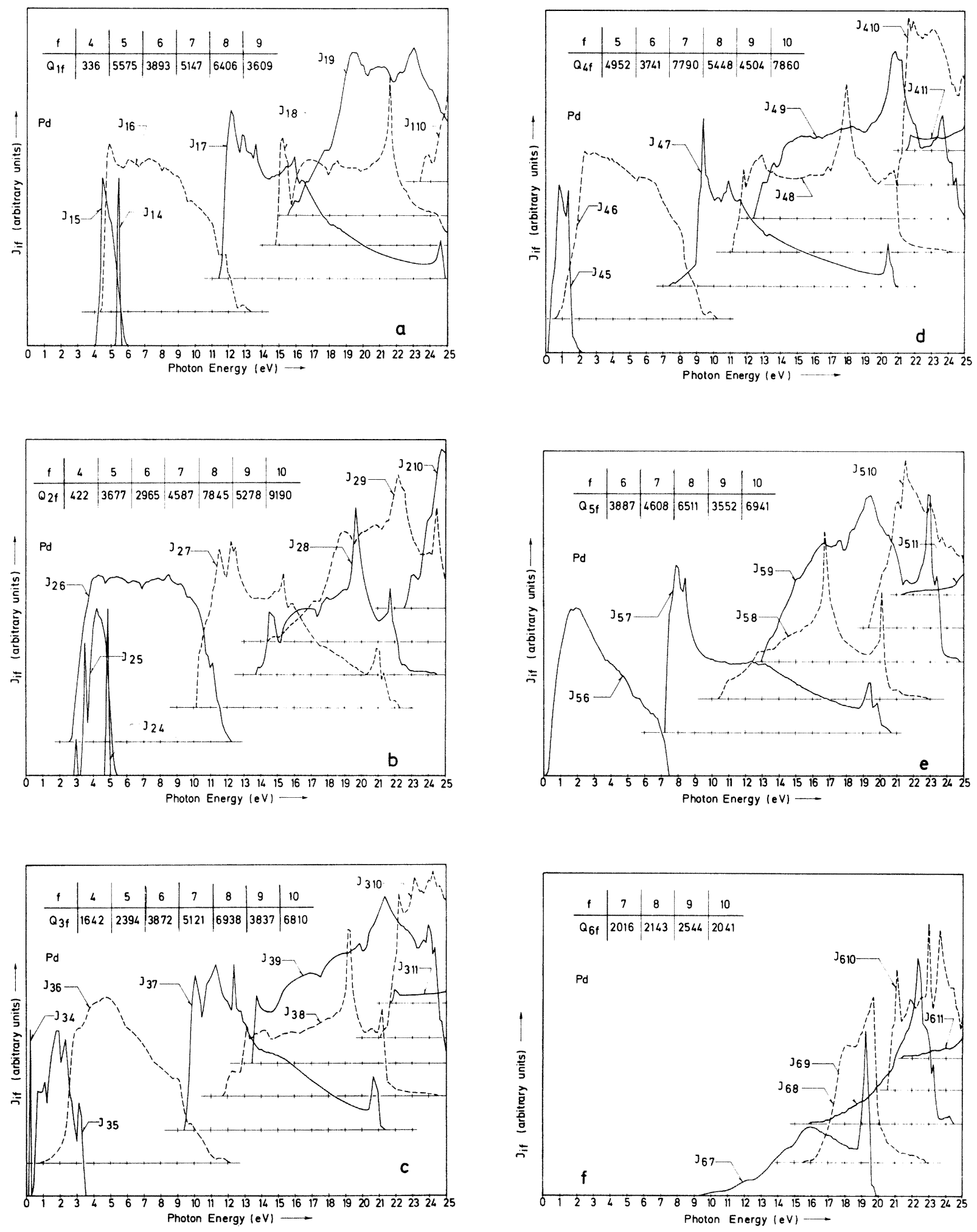

FIG. 4. (a)-(f) Partial joint density-of-states functions $J_{i f}(\hbar \omega)$ as defined in Eq. (1). All functions have been scaled to the same maximum value for convenience. The numbers $Q_{\text {if }}$ give, in arbitrary units, the maximum values, so that it is possible to estimate the relative intensities of the various $J_{\text {if }}$ functions. 


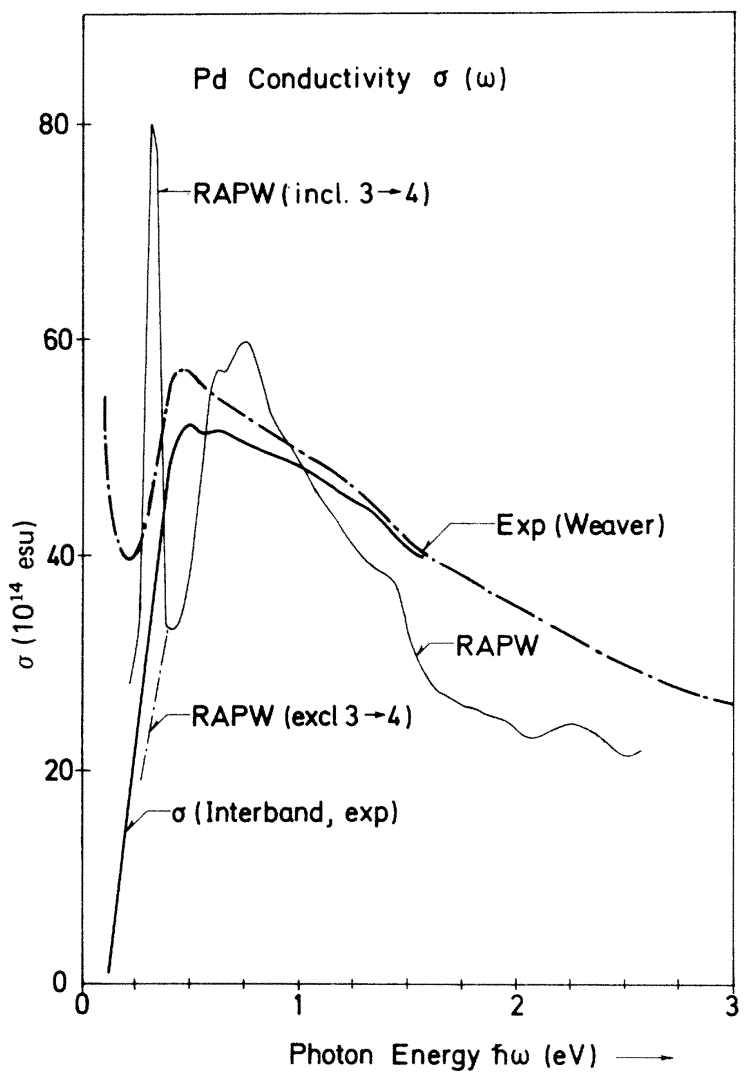

FIG. 5. Optical conductivity $\sigma(\omega)$ of palladium as measured by Weaver (Ref. 27) together with corresponding function derived from the present band-structure calculation.

$$
\begin{aligned}
N(E, \hbar \omega) \propto \sum_{i, f} \int & d \overrightarrow{\mathrm{k}}\left|M_{i f}\right|{ }^{2} P\left(\omega, \overrightarrow{\mathrm{k}}, E_{f}(\overrightarrow{\mathrm{k}})\right) \\
& \times \delta\left(E_{f}(\overrightarrow{\mathrm{k}})-E_{i}(\overrightarrow{\mathrm{k}})-\hbar \omega\right) \delta\left(E-E_{i}(\overrightarrow{\mathrm{k}})\right) \\
& \times f\left(E_{i}(\overrightarrow{\mathrm{k}})\right)\left[1-f\left(E_{f}(\overrightarrow{\mathrm{k}})\right)\right] .
\end{aligned}
$$

This function represents the number of electrons which are emitted when the photon energy is $\hbar \omega$ and which come from initial states with energy $E$. The quantity $M_{i f}$ is the momentum matrix element, whereas $P$ is an escape function including scattering effects for the excited electrons, and to some extent their interactions with the surface. Although the model at this level is very crude, we will simplify it further. As in the case of the calculation of the optical spectra it will be assumed that the matrix elements are constant, and the function $P$ will be replaced by an "effective" threshold function $C$ which can be taken outside the integral in (3). The function $C$, which is used here, is similar to the one constructed by Berglund and Spicer, ${ }^{31}$ and apart from a change in parameters it is identical to the function used in the calculations for silver. ${ }^{26}$ However, the approximate escape function was derived under the assumption that the final state bands are freeelectron-like which corresponds to a density-ofstates function without strong singularities.

The band structure of $\mathrm{Pd}$ does contain critical points in the final state regime and therefore it differs significantly from the free-electron model. Excited electrons which end up at critical points will be expected to contribute little to the emission spectra since their group velocity will be vanishingly small. Therefore we include an extra factor $v_{f}(\overrightarrow{\mathrm{k}})$, the final-state group speed, in the integrand of the expression for $N(E, \hbar \omega)$. Substituting the Fermi functions by step functions, our final expression for the energy distribution of emitted electrons is

$$
\begin{aligned}
N(E, \hbar \omega) \propto C \sum_{i, f} \int & d \overrightarrow{\mathrm{k}} v_{f}(\overrightarrow{\mathrm{k}}) \\
& \times \delta\left(E_{f}(\overrightarrow{\mathrm{k}})-E_{i}(\overrightarrow{\mathrm{k}})-\hbar \omega\right) \delta\left(E_{i}(\overrightarrow{\mathrm{k}})-E\right),
\end{aligned}
$$

where

$$
E_{i}(\overrightarrow{\mathrm{k}}) \leqslant E_{F} \text { and } E_{f}(\overrightarrow{\mathrm{k}}) \geqslant E_{F} .
$$

The escape function depends on the final-state energy $\left(E_{f}\right)$, electron mean free path $L\left(E_{f}\right)$, absorption coefficient $\alpha$, and the work function $e \phi$. The dependence of $L$ on $E_{f}$ was taken to be as in calculations for ${ }^{32} \mathrm{Rh}$ and it was adjusted to $10 \AA$ at $E_{f}-E_{F}=8 \mathrm{eV}$. The dependence on $\alpha$ is weak, and we used $\alpha=0.05 \AA^{-1}$ at all photon energies. The work function is $e \phi=5.4 \mathrm{eV}$. The energy distribution curves (EDC) as calculated from (4) for photon energies from 8 to $22 \mathrm{eV}$ are shown in Fig. 6. The spectra have been folded by a Lorentzian of $0.5 \mathrm{eV} \mathrm{FWHM} \mathrm{(full} \mathrm{width} \mathrm{at} \mathrm{half-maximum).}$ For a rough comparison to the measurements and calculations by Janak et al. ${ }^{17}$ in the photon energy range 7.7-11.6 eV we may consider our $10-\mathrm{eV}$ trace as representative. This EDC is seen (Fig. 6 ) to have peaks at $-0.2,-1.2,-2.2$, and $-3.6 \mathrm{eV}$. The corresponding experimental ${ }^{17}$ peak positions are $-0.15,-1.2,-2.2$, and $-3.5 \mathrm{eV}$ demonstrating excellent agreement between the calculation and experiment with respect to spectral position of elements of structure. This agreement encourages a more detailed comparison between the calculated and measured spectra. Four photon energies, $\hbar \omega=7.8,8.4,9.4$, and $10.2 \mathrm{eV}$ are selected for this examination, where we compare to the measurements by Traum and Smith. ${ }^{14}$ Figure 7 shows for $\hbar \omega=7.8 \mathrm{eV}$ the calculated energy distribution of joint density of states (EDJDOS) [ curve (a)], the calculated EDC (b), and its second derivative (c) together with three experimental traces. ${ }^{14}$ Curve 


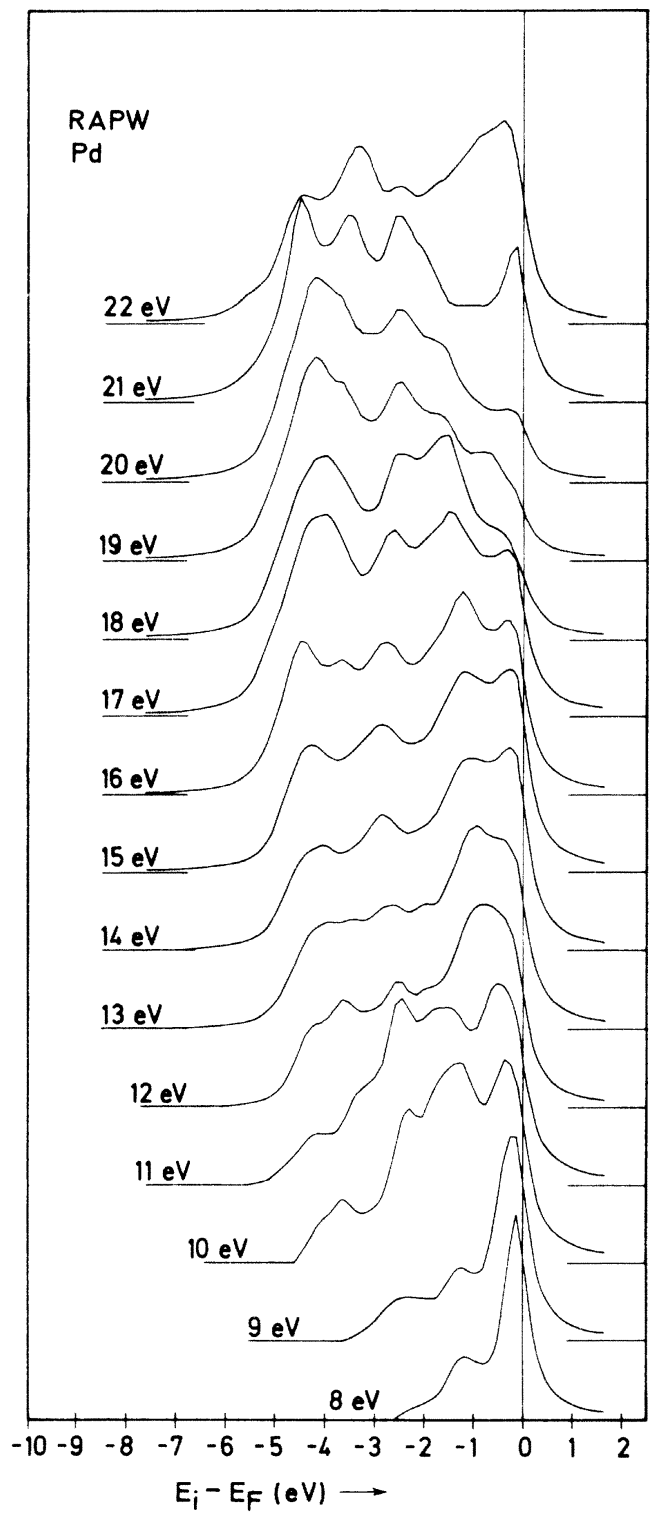

FIG. 6. Direct model energy distribution curves for Pd.

(e) is the EDC measured on clean Pd, whereas $f$ was taken after cesiation. The curve marked (d) represents the measured second derivative of the EDC, i.e., the third harmonic of the photocurrent. There has been made no attempt to scale the amplitudes, so that the vertical scales are different, and arbitrary, for all curves. Two strong peaks are observed at -0.15 and $-1.3 \mathrm{eV}$. The first one $(-0.15 \mathrm{eV})$ is due to transitions from band 4 and 5 to band 7, whereas $4 \rightarrow 6$ transitions are responsible for the second peak. The spectral positions of these peaks are identical in the experimental and theoretical traces. The apparent up-

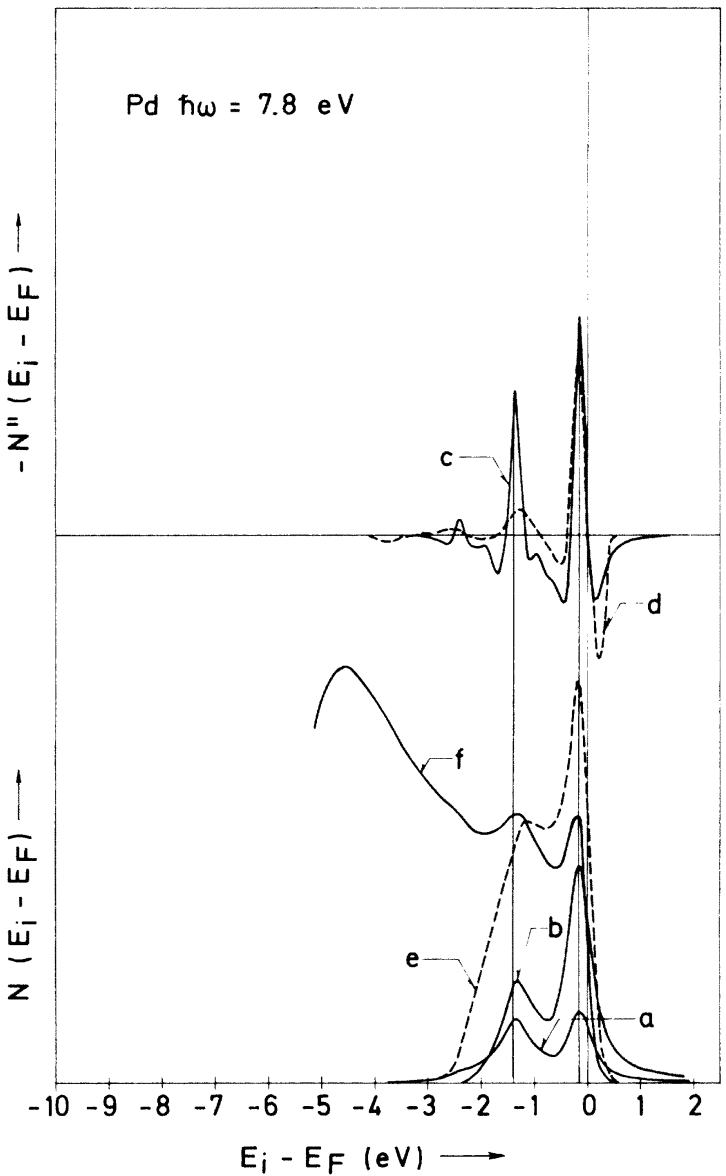

FIG. 7. Photoelectron spectra for Pd corresponding to 7.8-eV photons. (a) The calculated distribution of photoexcited electrons; (b) the calculated EDC; (c) the calculated second derivative; (e) the experimental trace (Ref. 14) obtained for a clean sample; (f) the EDC measured after cesiation; (d) the measured third harmonic of the photocurrent (Ref. 14). Vertical scales are arbitrary, and no attempt has been made to adjust amplitudes. The calculated EDJDOS and EDC functions are folded by a Lorentzian of $0.5 \mathrm{eV}$ FWHM.

ward shift of the second peak in curve (e) is considered as a line-shape effect due to the vacuum cutoff. A small third peak at $-2.5 \mathrm{eV}$ in the calculated spectra is also noticed in the spectrum taken after cesiation (f) and in the experimental second derivative $(\mathrm{d})$ at $-2.6 \mathrm{eV}$. This peak is due to $3 \rightarrow 6$ transitions. As will be demonstrated later, the regions in $k$ space where the transitions corresponding to a certain peak occur may be quite extended, all three peaks discussed above at least have important contributions from $k$ points at and close to the lines $Q$ and $\Lambda$. An increase of the photon energy $8.4 \mathrm{eV}$ (Fig. 8) produces only 
minor changes in the calculated EDJDOS and EDC [(a) and (b)]. The low-lying peak has split into two as can be seen from the calculated second derivative (c). The splitting is not seen in the experiment (d), but in the 9.4-eV spectrum (Fig. 9) it can be discerned $(-2.6$ and $-2.9 \mathrm{eV})$.

Further, at $9.4 \mathrm{eV}$ the leading peak has moved down to $-0.3 \mathrm{eV}$ in agreement with experiment. The position of the second peak, $-1.25 \mathrm{eV}$, is almost unchanged, and still matching the experiment. The calculated spectrum contains a strong peak at $-1.8 \mathrm{eV}$ which is not in the experimental trace, or at most it appears as a shoulder in $-N^{\prime \prime}$. The calculated peak is due to transitions from band 4 to 7 in an extremely small region around a $Q$ point where the spin-orbit coupling produces a singularity by splitting band 3 and 4 . The peak may well be exaggerated in the calculation because of slight inaccuracies in our $\vec{k}$-space inter-

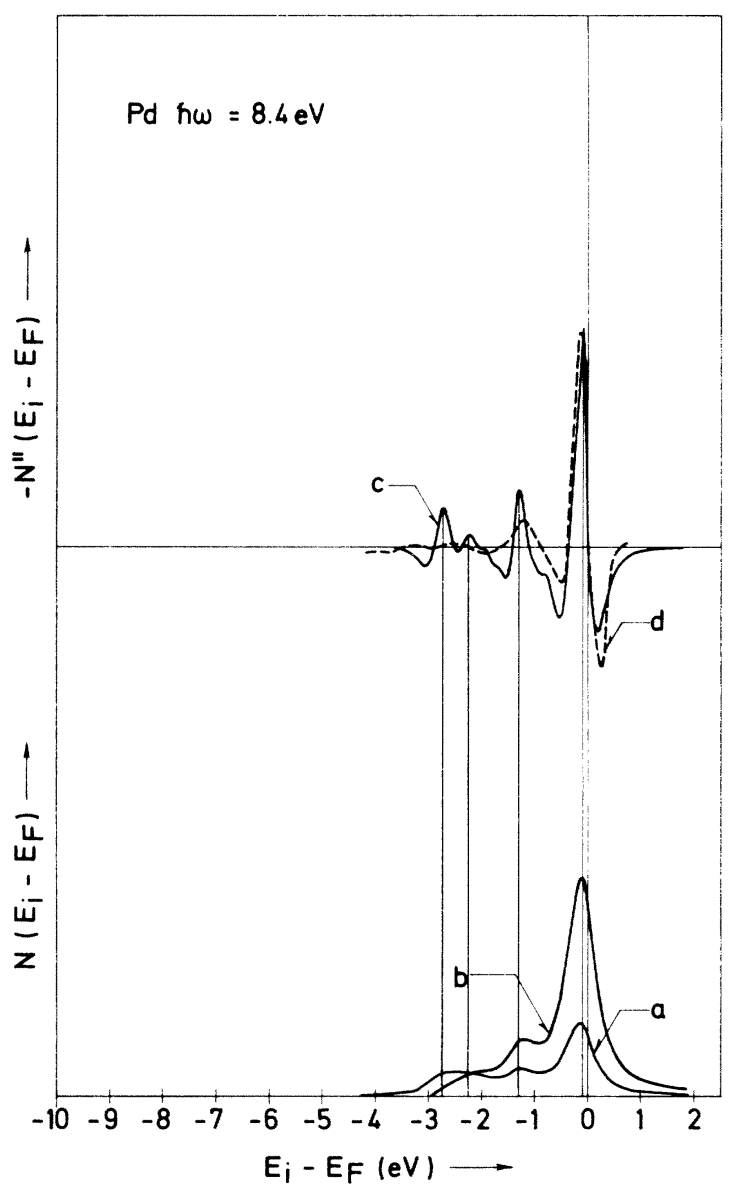

FIG. 8. Photoemission spectra for $\hbar \omega=8.4 \mathrm{eV}$. (a) Calculated EDJDOS; (b) calculated EDC; (c) calculated second derivative. The broken line (d) shows the experimental second derivative (Ref. 14). polation leading to band extrema which are not sufficiently sharp. Also at $\hbar \omega=10.2 \mathrm{eV}$ (Fig. 10) elements of structure appear in the calculated EDC, and in particular $N^{\prime \prime}$, which are not clearly present in the measured spectrum. Two such peaks in $-N^{\prime \prime}$ are found, one at $-1.77 \mathrm{eV}(3-7$ and $4 \rightarrow 7$ transitions) and another at $-3.1 \mathrm{eV}$. The latter is, however, very weak in the calculated EDC. The remaining elements of structure all have counterparts in the experiment and the spectral positions all agree. The calculated functions shown in the figure are broadened by $0.5 \mathrm{eV}$ (FWHM). The energy positions of peaks in the unbroadened functions are (excluding the two peaks discussed above) $-0.08,-0.25,-1.17,-2.33$, -3.63 , and $-5.16 \mathrm{eV}$. All these peaks have contributions from more or less extended regions in $\overrightarrow{\mathrm{k}}$ space. The first one $(-0.08 \mathrm{eV})$ is due to $6 \rightarrow 7$ transitions on a circle centered at a $\Lambda$ point $(1,1,1)(3 \pi / 4 a)$ and $5 \rightarrow 7$ transitions. The regions

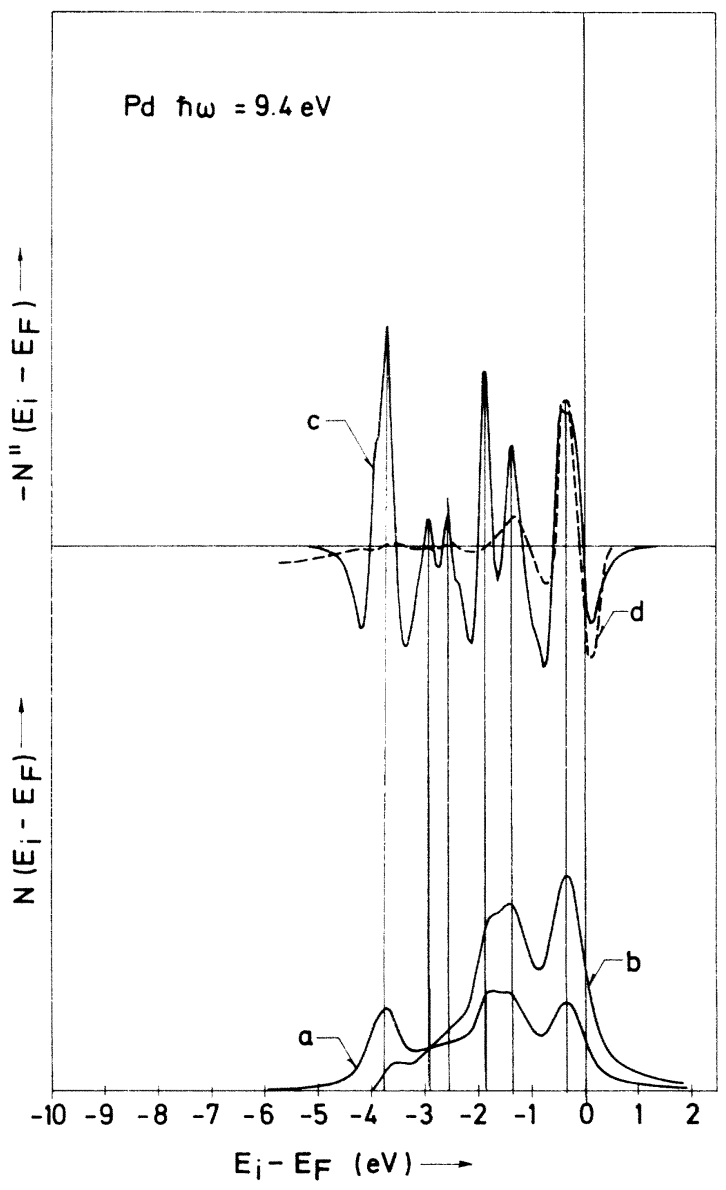

FIG. 9. Photoemission spectra at $\overline{\hbar \omega}=9.4 \mathrm{eV}$. The signatures (a)-(d) have the same meaning as in Fig. 8. 
in the Brillouin zone where the transitions occur are shown in the axiometric plot in Fig. 11. The second peak is entirely due to $5 \rightarrow 7$ transitions (Fig.12) The seventh band also contains all the final states for the peak at $-1.17 \mathrm{eV}$, the initial states being in the fourth band. The regions for these $4 \rightarrow 7$ transitions are shown in Fig. 13. The peak at $-2.33 \mathrm{eV}$ is very intense, and it is due to transitions over a very large region in the Brillouin zone (Fig. 14) from band 3 to band 7 . The final states for the last two peaks are all in band 6 . The $-3.63-\mathrm{eV}$ peak is due to $2 \rightarrow 6$ transitions in regions illustrated in Fig. 15, whereas $1 \rightarrow 6$ as well as $2-6$ transitions contribute to the peak at $-4.16 \mathrm{eV}$. The $2 \rightarrow 6$ transitions contributing to this peak are restricted to circular lines around the $(1,0,0)$ axes lying in planes perpendicular to these axes close to the square zone faces (Fig. 16).

The photon energy $\hbar \omega=10.2 \mathrm{eV}$ is particularly interesting since the calculated band structure

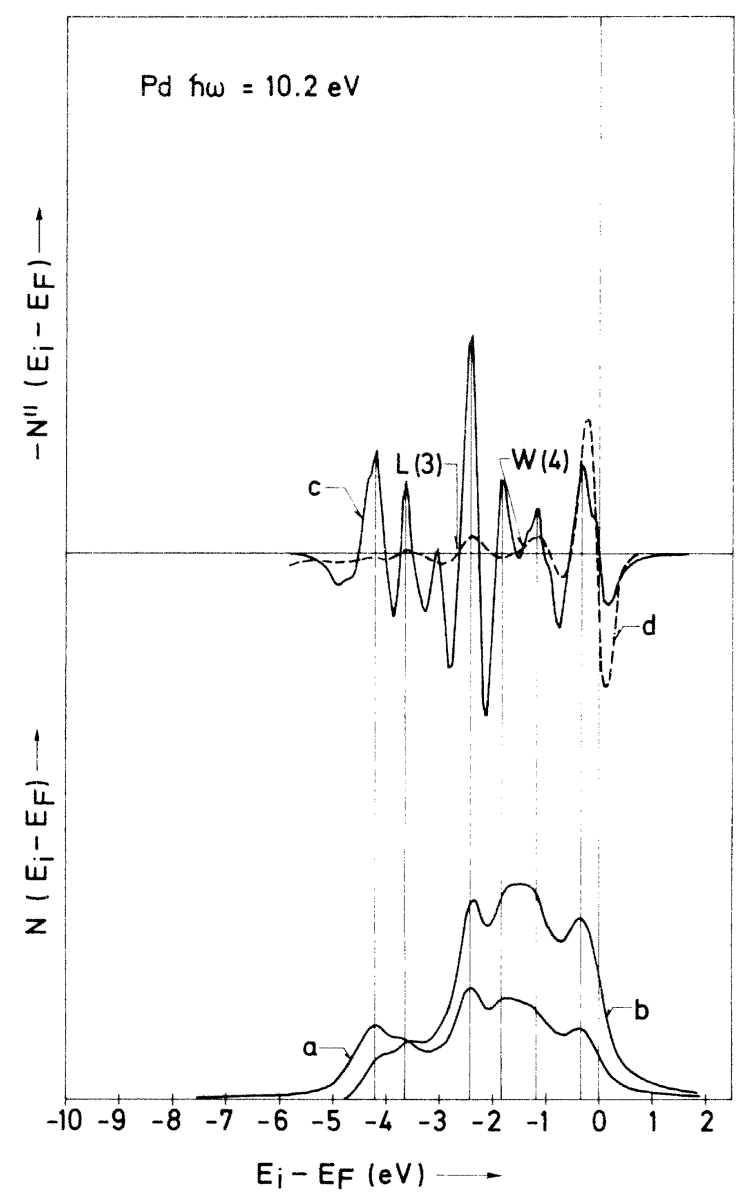

FIG. 10. Photoelectron spectra for $\hbar \omega=10.2 \mathrm{eV}$. The spectral positions of the initial-state energies in band 3 and 4 at $L$ and $W$ are indicated with arrows. contains two critical points, $3 \rightarrow 7$ at $L$ and $4 \rightarrow 7$ at $W$ with this energy gap. The initial states of these transitions are indicated in Fig. 10. It is obvious that these critical-point transitions are not placed in energy at peaks, but in this case rather where $N^{\prime \prime}$ is zero. The peaks in the EDC's cannot be related to transitions in specific single points, critical points, in the Brillouin zone but they have in general contributions from extended regions in $\vec{k}$ space.

\section{SUMMARY AND CONCLUSIONS}

The relativistic band-structure model $^{2}$ for palladium which agreed well with experiments concerning the Fermi surface has been extended to cover a large energy range and the entire Brillouin zone.

The band-structure calculation has been used in

$$
\begin{aligned}
& P d \hbar \omega=10.2 \mathrm{eV} \\
& E_{p}=-0.08 \mathrm{eV}
\end{aligned}
$$

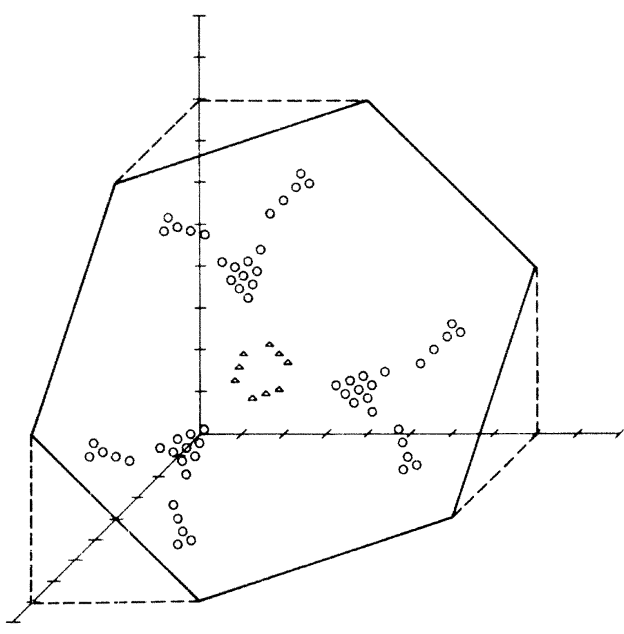

○: $5 \rightarrow 7$

$\triangle: 6 \rightarrow 7$

FIG. 11. Axiometric plot showing the $\vec{k}$-space regions contributing to the $-0.08-\mathrm{eV}$ peak in the EDC for $\bar{n} \omega=10.2$ $\mathrm{eV}$. The circles indicate the positions of the microcube centers from the Gilat-Raubenheimer integration where the energy of band 5 is $-0.08 \mathrm{eV}$ and the energy of band 7 is $-0.08 \mathrm{eV}+10.2 \mathrm{eV}$. The triangles define in the same way the loci of $6 \rightarrow 7$ transitions. 
Fif $n \omega=10.2 \mathrm{eV}$

$E_{p} \quad=-0.25 \mathrm{eV}$

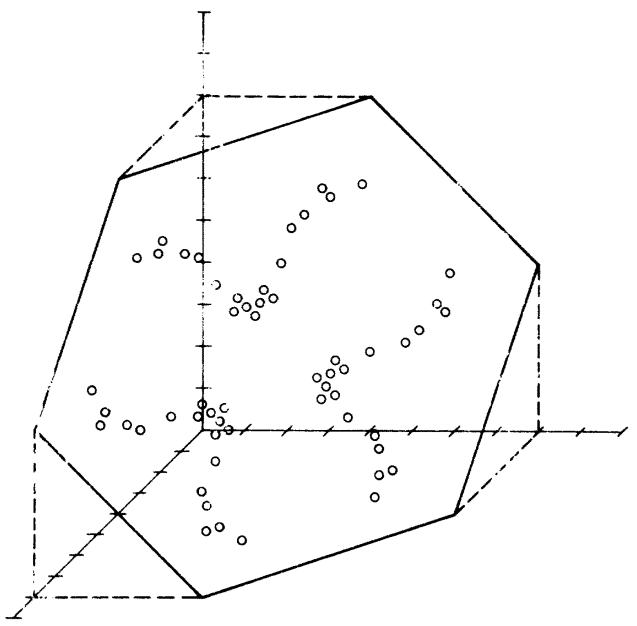

$\circ: 5 \rightarrow 7$

FIG. 12. Microcube centers for the transitions which take place from band 5 to band 7 and are giving rise to the peak $0.25 \mathrm{eV}$ below $E_{F}$ in the EDC for $\hbar \omega=10.2 \mathrm{eV}$.

Pd $\hbar \omega=10.2 \mathrm{eV}$

$E_{p}=-1.17 \mathrm{eV}$

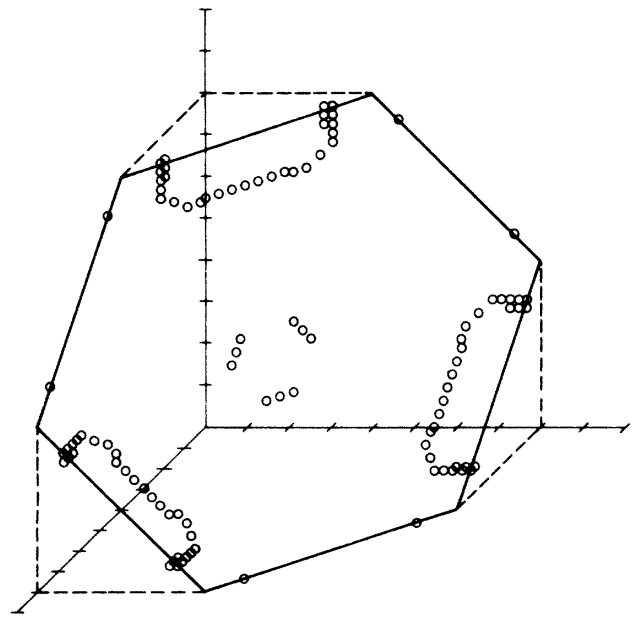

$\circ: 4 \rightarrow 7$

FIG. 13. Regions in $\vec{k}$ space where the transitions corresponding to the peak $1.17 \mathrm{eV}$ below $E_{F}$ in the 10.2-eV EDC.
$\mathrm{Pd} \hbar \omega=10.2 \mathrm{eV}$

$E_{p}=-2.33 \mathrm{eV}$

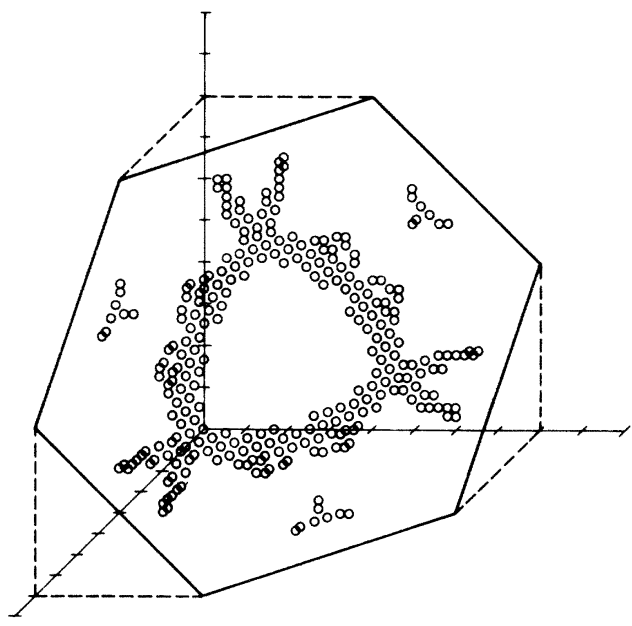

$\circ: 3 \rightarrow 7$

FIG. 14. Origin of the $3 \rightarrow 7$ transitions responsible for the peak $2.33 \mathrm{eV}$ below $E_{F}$ in the 10.2-eV EDC.

Pd $\hbar \omega=10.2 \mathrm{eV}$

$E_{p}=-3.63 \mathrm{eV}$

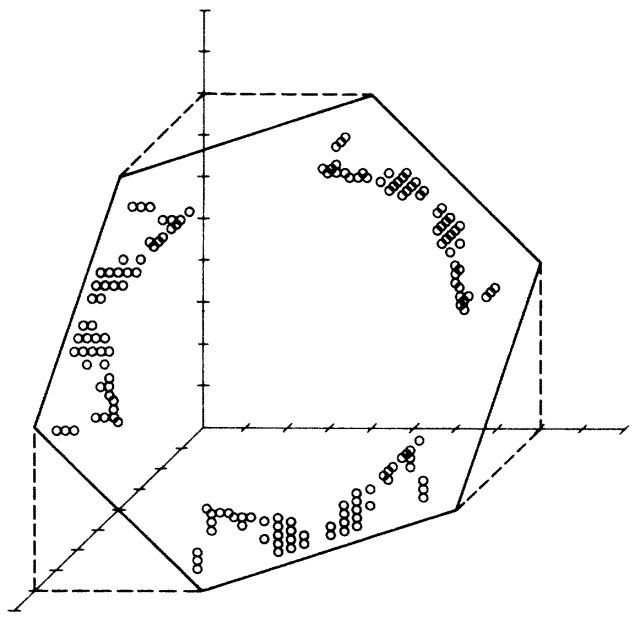

$\circ: 2 \rightarrow 6$

FIG. 15. Loci of $2 \rightarrow 6$ transitions for $\hbar \omega=10.2 \mathrm{eV}$ having initial-state energies $3.63 \mathrm{eV}$ below the Fermi level. 
$P d \hbar \omega=10.2 \mathrm{eV}$
$E_{p}=-4.16 \mathrm{eV}$

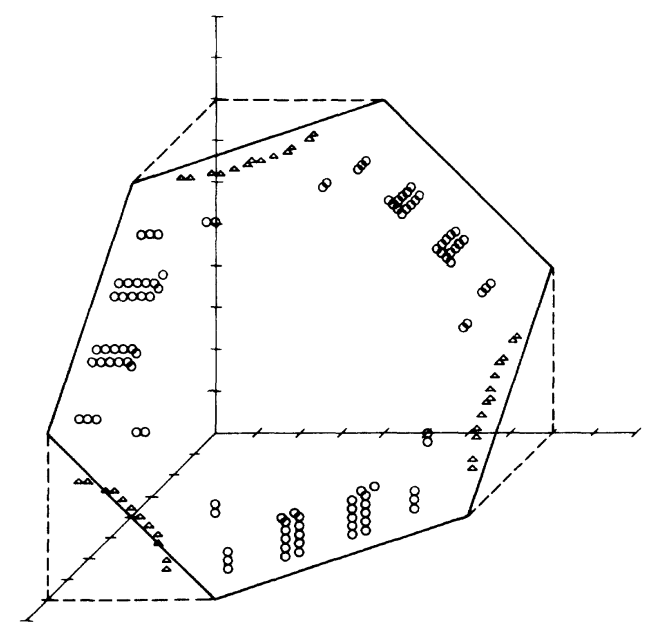

$\circ: 1 \rightarrow 6$

- : $2 \rightarrow 6$

FIG. 16. Triangles indicate the loci of $2 \rightarrow 6$ transitions and circles correspond to $1 \rightarrow 6$ transitions of energy $\hbar \omega=10.2 \mathrm{eV}$ and initial-state energy $4.16 \mathrm{eV}$ below $E_{F}$.

an examination of the optical properties of $\mathrm{Pd}$ in the uv regime by comparing calculated $E_{2}$ profiles and photoemission spectra to experimental results. The experimental $\epsilon_{2}(\omega)$ functions are quite difficult to obtain reliably so that independent measurements can reproduce the spectra since there are serious contamination problems. However, by comparing various experiments to our band calculation we suggest that the interband part of $\epsilon_{2}(\omega)$ for clean Pd should exhibit the following features. At low photon energies are two maxima at 0.5 and $1.1 \mathrm{eV}$ both due to transitions from band
4 to band 5. Five edgelike features appear at 3.0 , $3.3,3.7,4.4$, and $7.2 \mathrm{eV}$ corresponding to, respectively, $3 \rightarrow 6,2 \rightarrow 5,3 \rightarrow 5,1 \rightarrow 5$, and $5 \rightarrow 7$ transitions. The regions near $L, \Lambda$, and $Q$ points contribute essentially. The agreement between the calculation and the experiment was particularly informative in the case of the 7.2-eV structure since we from this could conclude that the calculated value of the seventh band at $L$ is reasonable, this level being very sensitive to the potential. Also the optical properties at high photon energies are largely influenced by the band structure around $L$ and the lines $\Lambda$ and $Q$. Structure at $9.7 \mathrm{eV}$ is due to $4 \rightarrow 7$ transitions whereas $1-7$ (around $L$ ) and $2 \rightarrow 7$ (around $W$ ) transitions are seen at $\hbar \omega \simeq 12.5 \mathrm{eV}$. It is suggested that a maximum in the experimental trace at $15.9 \mathrm{eV}$ can be related to transitions from band 1 to band 8 with an onset edge at $14.9 \mathrm{eV}$ at $W$. The elements of structure observed at 17.3 and $18.4 \mathrm{eV}$ are supposed to be due to $5 \rightarrow 8$ and $4 \rightarrow 8$ transitions. The $d \rightarrow f$ transitions at $\Gamma$ start at $19.0 \mathrm{eV}$ ac cording to the RAPW calculation and the calculated $\Gamma_{7}^{+} \rightarrow \Gamma_{6}^{-}$ gap is $20.2 \mathrm{eV}$, a value which fits the experiment nicely if it can be considered as the onset edge for transitions producing a clear maximum at $20.5 \mathrm{eV}$ in the observed profile.

Also the comparison between calculation and photoemission experiments demonstrated that consistent interpretation is possible. As in the case of the optical experiments it appeared that transitions in the region around $L$ and the symmetry lines $Q$ and $\Lambda$ play an important role. However, particular peaks in $\epsilon_{2}(\omega)$ as well as peaks in the EDC's cannot in general be related to transitions at singular points in the Brillouin zone, but are due to transitions in extended regions of $\overrightarrow{\mathrm{k}}$ space.

\section{ACKNOWLEDGMENTS}

The author wants to thank J. H. Weaver for communicating unpublished results and supplying valuable information. The advice from $\mathrm{O}$. K. Andersen and O. Jepsen is greatly appreciated.
${ }^{1}$ F. M. Mueller, A. J. Freeman, J. O. Dimmock, and A. M. Furdyna, Phys. Rev. B 1, 4617 (1970).

${ }^{2}$ O. K. Andersen, Phys. Rev. B $\underline{2}, 883$ (1970).

${ }^{3}$ F. M. Mueller, Phys. Rev. 153, 659 (1957).

${ }^{4}$ R. C. Vehse, E. T. Arakawa, and M. W. Williams, Phys. Rev. B 1, 517 (1970).

${ }^{5}$ A. Y.-C. Yu and W. E. Spicer, Phys. Rev. 169, 497 (1968).

${ }^{6}$ J. H. Weaver, Phys. Rev. B 11, 1516 (1975).

${ }^{7}$ Zh. Duisebaeva, M. I. Korsunskii, and G. P. Motulevich, Opt. Spektrosk. 34, 535 (1973) [Opt. Spectrosc. 34, 307
(1973)] .

${ }^{8}$ G. A. Bolotin, M. M. Kirillova, L. V. Nomerovannaya, and M. M. Noskov, Fiz. Met. Mettalloved. 23, 463 (1967).

${ }^{9}$ B. Dold and R. Mecke, Optik (Stuttg.) 22, 453 (1965).

${ }^{10} \mathrm{P}$. Lostis, J. Phys. (Paris) 25, 118 (1964).

${ }^{11}$ P. B. Johnson and R. W. Christy, Phys. Rev. B $\underline{9}$, 5056 (1974).

${ }^{12}$ L. Hodges, H. Ehrenreich, and N. D. Lang, Phys. Rev. 152 (1966); H. Ehrenreich and L. Hodges, in Methods in Computational Physics, edited by B. Alder, S. Fern- 
bach, and M. Rotenberg (Academic, New York, 1968), Vol. 8, p. 149.

${ }^{13}$ N. V. Smith and L. F. Mattheiss, Phys. Rev. B 9,1341 (1974).

${ }^{14}$ M. M. Traum and N. V. Smith, Phys. Rev. B $\underline{9}, 1353$ (1974).

${ }^{15}$ N. V. Smith, Phys. Rev. B 9, 1365 (1974).

${ }^{16}$ N. E. Christensen, Phys. Status Solidi B 52, 241 (1972).

${ }^{17} \mathrm{~J}$. F. Janak, D. E. Eastman, and A. R. Williams, Solid State Commun. $\underline{8}, 271$ (1970).

${ }^{18} \mathrm{~B}$. Segall and F. S. Ham, in Methods in Computational Physics (Academic, New York, 1968), Vol. 8.

${ }^{19} \mathrm{D}$. Liberman, J. T. Waber, and D. T. Cromer, Phys. Rev. 137, A27 (1965).

${ }^{20}$ G. S. Painter, J. S. Faulkner, and G. M. Stocks, Phys. Rev. B 9, 2448 (1974).

${ }^{21}$ N. E. Chrristensen and B. O. Seraphin, Phys. Rev. B $\underline{4}$, 3321 (1971).
${ }^{22}$ G. Gilat and L. J. Raubenheimer, Phys. Rev. 144, 390 (1966).

${ }^{23} \mathrm{~F}$. Herman and S. Skillman, Atomic Structure Calculations (Prentice-Hall, Englewood Cliffs, N. J., 1963).

${ }^{24}$ D. B. B. Rijsenbrij and J. M. Fondse, Solid State Commun. 17, 1081 (1975).

${ }^{25}$ N. E. Christensen, Phys. Status Solidi 31, 635 (1969).

${ }^{26}$ N. E. Christensen, Phys. Status Solidi $\bar{B} 54,551$ (1972).

${ }^{27} \mathrm{~J}$. H. Weaver (private communication).

${ }^{28}$ N. E. Christensen, Phys. Rev. B 13, 2698 (1976).

${ }^{29}$ N. E. Christensen and B. Feuerbacher, Phys. Rev. B 10,2349 (1975).

${ }^{30}$ B. Feuerbacher and N. E. Christensen, Phys. Rev. B 10, 2373 (1975).

${ }^{31}$ C. N. Berglund and W. E. Spicer, Phys. Rev. 136, A1030 (1964).

${ }^{32}$ N. E. Christensen, Phys. Status Solidi 55, 117 (1973). 